Check for updates

Cite this: RSC Adv., 2019, 9, 14072

Received 20th March 2019

Accepted 11th April 2019

DOI: 10.1039/c9ra02146a

rsc.li/rsc-advances

\section{Strain sensitivity of band structure and electron mobility in perovskite $\mathrm{BaSnO}_{3}$ : first-principles calculation}

\author{
Yaqin Wang, (D) *ab Runqing Sui, ${ }^{a}$ Mei $\mathrm{Bi}^{\mathrm{a}}{ }^{\mathrm{W}}$ Wu Tang ${ }^{\mathrm{b}}$ and Sude $\mathrm{Ma}^{\mathrm{a}}$
}

A first-principles electronic structure calculation is utilized to contrastively investigate the crystal structure, band structure, electron effective mass and mobility of perovskite $\mathrm{BaSnO}_{3}$ under hydrostatic and biaxial strain. Strain-induced changes in relative properties are remarkable and more sensitive to hydrostatic strain than biaxial strain. The structure of $\mathrm{BaSnO}_{3}$ remains cubic under hydrostatic strain, while it becomes tetragonal under biaxial strain. Originating from the strain sensitivity of the $\mathrm{Sn} 5 \mathrm{~s}$ orbitals in the conduction band minimum, the band gaps of $\mathrm{BaSnO}_{3}$ decrease for both types of strain from $-3 \%$ to $3 \%$. $\mathrm{BaSnO}_{3}$ under tensile hydrostatic strain exhibits higher electron mobility than it does under tensile biaxial strain because of the smaller electron effective mass in the corresponding strain. In contrast, the opposite phenomenon exists in compressive strain. Our results demonstrate that strain could be an alternative way to modify the band gap and electron mobility of $\mathrm{BaSnO}_{3}$.

\section{Introduction}

Recently perovskite-type oxide $\mathrm{BaSnO}_{3}$ has become a research hotspot, due to its high electron mobility and visible light transmittance at room temperature, as well as thermal stability. ${ }^{1}$ The cubic $\mathrm{BaSnO}_{3}$ with a band gap of $3.1 \mathrm{eV}$ is stable at temperatures up to $1000{ }^{\circ} \mathrm{C}^{2}$ Its high visible optical transparency makes it is commonly used as a transparent electrode material or as electronic components for power devices, and it is regarded as a good substitute material for the traditional transparent conductive oxides. On the other hand, due to the Sn 5 s orbitals on the bottom of the conduction band, $\mathrm{BaSnO}_{3}$ has a high electron mobility at room temperature, with values of 320 $\mathrm{cm}^{2} \mathrm{~V}^{-1} \mathrm{~s}^{-1}$ (ref. 3) and $150 \mathrm{~cm}^{2} \mathrm{~V}^{-1} \mathrm{~s}^{-1}$ (ref. 4) for single crystal and film structures, respectively. These properties suggest that $\mathrm{BaSnO}_{3}$ is an ideal channel material in perovskite heterostructures, compared with the traditional $\mathrm{SrTiO}_{3}$ thin films. In the past 50 years, the best electron mobility and conductivity of $\mathrm{SrTiO}_{3}$ thin film has been $10 \mathrm{~cm}^{2} \mathrm{~V}^{-1} \mathrm{~s}^{-1}$ and $500 \mathrm{~S} \mathrm{~cm}^{-1}$ at room temperature ${ }^{1}$ resulting in the related interesting properties of the $\mathrm{LaAlO}_{3} / \mathrm{SrTiO}_{3}$ heterostructure being limited to low temperatures. ${ }^{5}$ The appearance of $\mathrm{BaSnO}_{3}$ is expected to make the quantum effect appear in the perovskite heterostructure at room temperature, expanding the application ranges of perovskite electronic devices.

${ }^{a}$ Key Laboratory of Fluid and Power Machinery, School of Material Science and Engineering, Xihua University, Chengdu, 610039, China

${ }^{b}$ State Key Laboratory of Electronic Thin Films and Integrated Devices, University of Electronic Science and Technology of China, Chengdu, 610054, China. E-mail: wangyqyyxf@sina.com
To date, previous studies have used many methods to modify the band gap and electron mobility of $\mathrm{BaSnO}_{3}$ thin films. The typical method is "Doping engineering" with La into the Ba site and $\mathrm{Sb}$ into the $\mathrm{Sn}$ site. The band gap can be effectively adjusted from $3.48 \mathrm{eV}$ to $4.23 \mathrm{eV} .^{2,6-8}$ However, the electron mobility can only reach $70-150 \mathrm{~cm}^{2} \mathrm{~V}^{-1} \mathrm{~s}^{-1}$ at room temperature. In the earliest work, the electron mobility of (Ba, La) $\mathrm{SnO}_{3}$ thin films on $\mathrm{SrTiO}_{3}$ (001) substrate obtained by Kim et al. ${ }^{3}$ was $70 \mathrm{~cm}^{2} \mathrm{~V}^{-1}$ $\mathrm{s}^{-1}$ at a doping level of $4 \times 10^{20} \mathrm{~cm}^{-3}$. Kookrin Char et al. ${ }^{9}$ and Shiogai et al. ${ }^{\mathbf{1 0}}$ improved the electron mobility of $\mathrm{La}-\mathrm{BaSnO}_{3}$ thin films to $80 \mathrm{~cm}^{2} \mathrm{~V}^{-1} \mathrm{~s}^{-1}$ and $78 \mathrm{~cm}^{2} \mathrm{~V}^{-1} \mathrm{~s}^{-1}$ by depositing the $\mathrm{BaSnO}_{3}$ and (Ba, Sr) $\mathrm{SnO}_{3}$ buffer layers on $\mathrm{SrTiO}_{3}(001)$ substrate, respectively. K. H. Kim et al. ${ }^{11}$ has reported that using single crystal $\mathrm{BaSnO}_{3}$ (001) as substrate can reduce the appearance of dislocation in $\mathrm{BaSnO}_{3}$ thin films, increasing the electron mobility up to $102 \mathrm{~cm}^{2} \mathrm{~V}^{-1} \mathrm{~s}^{-1}$. Bharat Jalan et al., ${ }^{1}$ Lebens Higgins et al., ${ }^{12}$ and Susanne Stemmer et al. ${ }^{4}$ have deposited $\mathrm{La}-\mathrm{BaSnO}_{3}$ thin films with ultra-high crystallinity by molecular beam epitaxy, improving the electron mobility to 120 $\mathrm{cm}^{2} \mathrm{~V}^{-1} \mathrm{~s}^{-1}, 81 \mathrm{~cm}^{2} \mathrm{~V}^{-1} \mathrm{~s}^{-1}$, and $150 \mathrm{~cm}^{2} \mathrm{~V}^{-1} \mathrm{~s}^{-1}$, respectively. All these studies indicate that due to the ionized impurities scattering caused by the $\mathrm{La}(\mathrm{Sb})$ atom doping in the $\mathrm{La}(\mathrm{Sb})-$ $\mathrm{BaSnO}_{3}$ thin films, the electron mobilities of $\mathrm{La}(\mathrm{Sb})-\mathrm{BaSnO}_{3}$ are limited. So far, the highest electron mobility of $\mathrm{BaSnO}_{3}$ film obtained at room temperature is $150 \mathrm{~cm}^{2} \mathrm{~V}^{-1} \mathrm{~s}^{-1}$. Thus, other methods to tune the band gap and electron mobility of $\mathrm{BaSnO}_{3}$ are needed.

The method of "Strain engineering" is a widely used method to obtain desired properties of materials. Many studies have indicated that external stresses such as hydrostatic pressure and internal strains from lattice and thermal mismatch can 
alter the structure and electronic properties of semiconductors. The band type of ultrathin [0001] ZnO nanowire changes from direct to indirect under uniaxial compressive strain, along with the structural transition from wurtzite to hexagonal. ${ }^{13}$ The band gaps of $\mathrm{Sn}$ oxides with $\mathrm{SnO}_{6}$ octahedra decrease (increase) by 0.8-1.0 eV with a hydrostatic strain of $2 \%(-2 \%)$, showing that they are highly sensitive to hydrostatic strain. ${ }^{\mathbf{1 4}}$ The tensile biaxial strain from the substrate destroys the two-dimensional electron gas (2DEG) at the $\mathrm{LaAlO}_{3} / \mathrm{SrTiO}_{3}$ (001) interface, while the compressive biaxial strain induces the 2DEG and increases the critical thickness of $\mathrm{LaAlO}_{3}$ thin films for the insulator-tometal transition..$^{15,16}$ Also, the tensile biaxial strain is experimentally demonstrated to be effective at reducing the mobility of the 2DEG at the $\mathrm{LaAlO}_{3} / \mathrm{SrTiO}_{3}(001)$ interface. ${ }^{\mathbf{1 6}}$ The epitaxial strain dependence of the spontaneous polarizations and Curie temperatures varies considerably for different ferroelectric thin film systems, such as $\mathrm{BaTiO}_{3}, \mathrm{PbTiO}_{3}, \mathrm{LiNbO}_{3}$, and $\mathrm{BiFeO}_{3} \cdot{ }^{17-20}$

To tune the electronic properties of $\mathrm{BaSnO}_{3}$ through epitaxial in-plane misfit strains and external hydrostatic pressure, we have used first-principles calculations to demonstrate a detailed study of strain engineering of $\mathrm{BaSnO}_{3}$. The changes in crystal structure, electronic structure, electron effective mass and mobility due to the imposed strain have been investigated. Some commercially available substrate materials have also been concluded in our work.

\section{Methods and models}

In this work, all the density functional theory (DFT) simulations were performed using the Vienna Ab initio Simulation Package (VASP). ${ }^{21}$ The electron-ion interactions were described by the projector augmented-wave (PAW) ${ }^{22}$ pseudopotentials method. A cutoff energy of $440 \mathrm{eV}$ for the plane-wave basis set, and a Monkhorst-Pack $k$-point mesh of $6 \times 6 \times 6$ for the unit cell of $\mathrm{BaSnO}_{3}$ was employed to get well-converged results. The force tolerance on every atom was smaller than $0.01 \mathrm{eV} \AA^{-1}$. For the electronic exchange-correlation interactions, the Heyd-Scuseria-Ernzerhof (HSE06) ${ }^{23}$ hybrid functional theory was used, and $43 \%$ contribution of Hartree-Fock (HF) exchange in HSE06 functional theory was set to obtain the accurate band gap of $\mathrm{BaSnO}_{3}$.

A cubic perovskite $\mathrm{BaSnO}_{3}$ unit cell was used to model the variations of properties under hydrostatic strain $\left(\varepsilon_{x x}=\varepsilon_{y y}=\varepsilon_{z z}\right)$ and biaxial strain $\left(\varepsilon_{x x}=\varepsilon_{y y} \neq \varepsilon_{z z}\right)$ from $-3 \%$ to $3 \%$, at intervals of $1 \%$. The relaxed lattice constant of $4.129 \AA$ from HSE06 theory was used to calculate the properties of unstrained $\mathrm{BaSnO}_{3}$. The lattice parameters along the $x, y$, and $z$-axes were adjusted to simulate various applied hydrostatic strains from $-3 \%$ to $3 \%$, and allowing the atoms to fully relax. For the case of biaxial strain, the lattice parameters along the $x$ and $y$-axes were changed equally, and the structure was allowed to relax in the $z$ axial direction. The electron effective mass was determined by analyzing the calculated band structures at the energy band extremum using the standard equation ${ }^{24}$

$$
\frac{1}{m_{i}}=\frac{1}{\hbar^{2}} \frac{\partial^{2}}{\partial k^{2}} E_{\text {cond }}\left(k_{\mathrm{CBM}}\right)
$$

where $i(\|, \perp)$ represents the direction of the calculated electron effective mass in Fig. 1.

\section{Discussion and results}

Strain-free $\mathrm{BaSnO}_{3}$ has a cubic perovskite structure and belongs to the $P m \overline{3} m$ space group. It is composed of a corner-sharing $\mathrm{SnO}_{6}$ octahedron, with a $\mathrm{Ba}^{2+}$ filling in the structure. For the case of hydrostatic strain $\left(\varepsilon_{x x}=\varepsilon_{y y}=\varepsilon_{z z}\right), \mathrm{BaSnO}_{3}$ remains cubic [Fig. 1(a)] and is the same as strain-free $\mathrm{BaSnO}_{3}$. However, when biaxial strain $\left(\varepsilon_{x x}=\varepsilon_{y y} \neq \varepsilon_{z z}\right)$ is imposed, the symmetry is changed and the crystal structure becomes tetragonal [Fig. 1(f)]. Along with the different structure changes between biaxial and hydrostatic strain, $\mathrm{BaSnO}_{3}$ also presents discrepant electronic properties. Fig. 1(c), (d) and (e) show typical band structures of $\mathrm{BaSnO}_{3}$ under $-3 \%$ hydrostatic strain, 0 strain and $-3 \%$ biaxial strain, respectively. The results show that $\mathrm{BaSnO}_{3}$ displays indirect band gaps in both strain and strain-free structures. The conduction band minimum (CBM) under biaxial and hydrostatic strain both occurs at the $\Gamma$ point. However, originating from the different structural symmetry of $\mathrm{BaSnO}_{3}$ under biaxial and hydrostatic strain, the valence band maximum (VBM) appears at the $R$ point under biaxial strain and at the $A$ point under hydrostatic strain. The electron effective mass is determined by analyzing the calculated band structure near $\Gamma$ along the $z$-axial direction in reciprocal space $\left(m_{\|}\right)$and along the $x$ axial or $y$-axial directions $\left(m_{\perp}\right.$, perpendicular to the $z$-axial direction), as indicated in Fig. 1(b) and (g). According to the symmetry, it can be obtained that the electron effective masses in the $x, y$, and $z$-axials are equal under hydrostatic strain $\left(m_{\|}=\right.$ $\left.m_{\perp}\right)$, while they are shown to be different under biaxial strain $\left(m_{\|} \neq m_{\perp}\right)$. The accurate values are presented in the following content.

In order to clearly present structural changes of $\mathrm{BaSnO}_{3}$ under different strains, the lattice constants, bond lengths and volumes of $\mathrm{BaSnO}_{3}$ under different biaxial and hydrostatic strain are shown in Fig. 2. The lattice parameter of strain-free cubic $\mathrm{BaSnO}_{3}$ is $4.129 \AA$ from HSE calculation, in agreement with the experimental value of $4.115 \AA^{.25}$ The in-plane lattice constant $a$ and bond length $d_{a}(=a / 2)$ increase with increasing biaxial strain from compressive strain to tensile strain. The outof-plane lattice constant $c$ and bond length $d_{c}(=c / 2)$ decrease with the biaxial strain, resulting from the Poisson effect. For the case of hydrostatic strain, $\mathrm{BaSnO}_{3}$ remains cubic, and the change of lattice constants $(a=c)$ and bond lengths $\left(d_{a}=d_{c}\right)$ is the same as the shift of $a$ and $d_{a}$ under biaxial strain, that is, an increase with hydrostatic strain from compressive strain to tensile strain. As a result, the volumes of $\mathrm{BaSnO}_{3}$ under hydrostatic strain shift significantly toward higher values than that under biaxial strain. The volume of $\mathrm{BaSnO}_{3}$ is $80.13 \AA^{3}$ under $3 \%$ hydrostatic strain, which is about $4 \AA^{3}$ larger than that of $\mathrm{BaSnO}_{3}$ with the same biaxial strain.

Next, we carried out comparison studies for the electronic properties of $\mathrm{BaSnO}_{3}$ under different strain. Table 1 lists the lattice and electronic parameters of strain-free $\mathrm{BaSnO}_{3}$. The band gap is obviously underestimated to be around $0.325 \mathrm{eV}$ from the GGA calculation. The value from HSE calculation is 
(c)

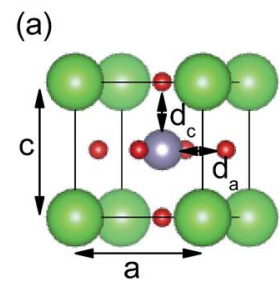

(b)

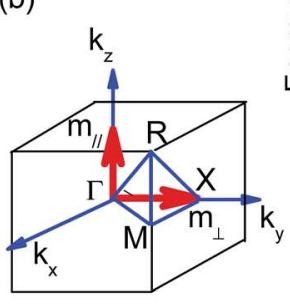

$-3 \%$ Hydrostatic Strain (d)

(e)

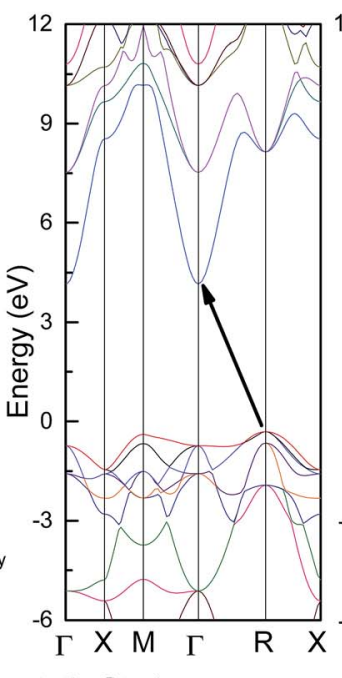

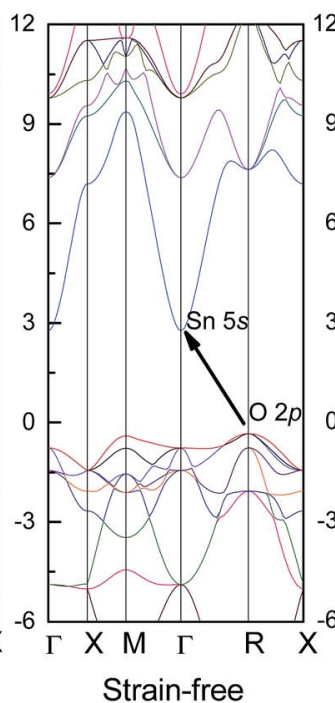

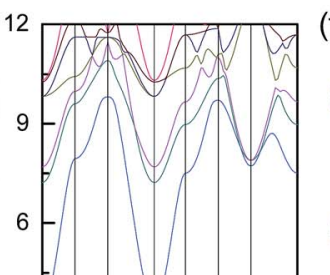

(f)

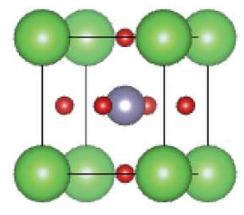

(g)

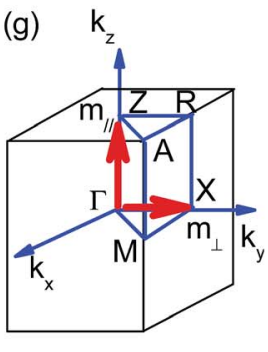

\section{$-3 \%$ Biaxial Strain}

Fig. 1 (a and f) are the $\mathrm{BaSnO}_{3}$ unit cell under $-3 \%$ hydrostatic strain (cubic structure) and $-3 \%$ biaxial strain (tetragonal structure). The green, gray and red circles represent $\mathrm{Ba}, \mathrm{Sn}$ and $\mathrm{O}$ atoms, respectively. $a$ and $c$ are the lattice constants of the $\mathrm{BaSnO}_{3}$ unit cell. $d_{a}$ and $d_{c}$ are the distances between $\mathrm{Sn}$ and $\mathrm{O}$ atoms in horizontal and vertical planes, respectively. (c, $\mathrm{d}$ and $\mathrm{e}$ ) are the calculated band structures of $\mathrm{BaSnO}_{3}$ under $-3 \%$ hydrostatic strain, 0 strain and $-3 \%$ biaxial strain, respectively. ( $\mathrm{b}$ and $\mathrm{g}$ ) are the Brillouin zones of the cubic structure and tetragonal structure indicating high-symmetry directions. Red arrows show the direction along which the electron effective masses are calculated.

$3.091 \mathrm{eV}$, consistent with the experimental values of $3.1 \mathrm{eV} .^{28}$ Fig. 3 shows the relationships between the calculated band gap from HSE calculation and biaxial strain or hydrostatic strain. The band gap of $\mathrm{BaSnO}_{3}$ decreases with increasing strain from compressive strain to tensile strain for both biaxial and hydrostatic strain. With an increase in biaxial and hydrostatic strain from $-3 \%$ to $3 \%$, the values drop from $3.70 \mathrm{eV}$ to $2.51 \mathrm{eV}$, and $4.46 \mathrm{eV}$ to $1.89 \mathrm{eV}$, respectively. The linear relationships under biaxial strain and hydrostatic strain, respectively, can be expressed as follows:

$$
E_{\mathrm{g}, \mathrm{bi}}=3.096-19.69 \varepsilon_{x x}
$$
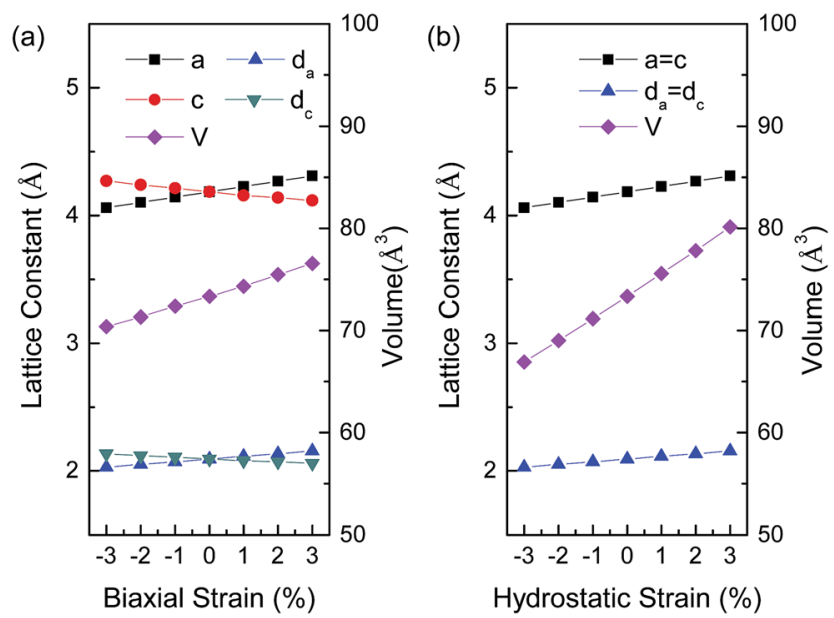

Fig. 2 Lattice constants, bond lengths and volumes of the $\mathrm{BaSnO}_{3}$ unit cell as a function of (a) biaxial strain, and (b) hydrostatic strain. The corresponding lattices and bonds are marked in Fig. 1(a).

$$
E_{\mathrm{g}, \mathrm{hyd}}=3.129-42.72 \varepsilon_{x x}
$$

To evaluate the change of band gap with strain, we further calculated the deformation potentials, which can determine the quality of device modeling ${ }^{33}$ and are difficult to obtain from experiment. ${ }^{34}$ The sum deformation potential under in-plane strain can be calculated by the following equation: ${ }^{33}$

$$
\Delta E_{\mathrm{g}}{ }^{X}=D_{\perp}{ }^{X}\left(\varepsilon_{x x}+\varepsilon_{y y}\right)
$$

where $X$ represents $T-R$ and $\Gamma-\Gamma$ for $\mathrm{BaSnO}_{3}$ under biaxial strain, and $\Gamma-A$ and $\Gamma-\Gamma$ for hydrostatic strain. Table 2 lists the calculated deformation potentials, which are important for $\mathrm{BaSnO}_{3}$ to predict the band positions under realistic strain.

To further explain the change of band gap versus strain, we plotted the energy of the CBM and VBM as a function of strain in Fig. 3. The variation tendency of the CBM is more remarkable than that of the VBM under both biaxial and hydrostatic strain. The $\mathrm{CBM}$ of $\mathrm{BaSnO}_{3}$ is mainly comprised of $\mathrm{Sn} 5 \mathrm{~s}$ orbitals, while the VBM consists of $\mathrm{O} 2 \mathrm{p}$ orbitals. The results thus indicate that the Sn $5 \mathrm{~s}$ orbitals play a major role in the strain sensitivity of the band gap in $\mathrm{BaSnO}_{3}$.

In addition to the band structure, we also calculated the electron effective mass for the conduction band minimum at the $\Gamma$ point along the $x, y$ and $z$-axial directions in reciprocal space. The calculated longitudinal and transverse effective masses at the $\Gamma$ point in strained $\mathrm{BaSnO}_{3}$ are shown in Fig. 4 . The electron effective mass of $\mathrm{BaSnO}_{3}$ changes remarkably with the strain from $-3 \%$ to $3 \%$. For the case of biaxial strain, the transverse and longitudinal electron effective masses both 
Table 1 The lattice parameters $a$, indirect band gap $E_{g}^{\text {ind }}$, and electron effective mass $m_{\mathrm{e}}^{*}$ for strain-free $\mathrm{BaSnO}_{3}$ calculated by GGA and $\mathrm{HSEO} 6$ methods, compared to the available data

\begin{tabular}{llllll}
\hline & GGA & HSE06 & Exp. & GGA-Ref. & HSE06-Ref. \\
\hline$a(\AA)$ & 4.186 & 4.129 & 4.115 (ref. 25) & 4.142 (ref. 26) & 4.13 (ref. 27) \\
$E_{\mathrm{g}}^{\text {ind }}(\mathrm{eV})$ & 0.325 & 3.091 & 3.1 (ref. 28) & 0.74 (ref. 26) & 3.22 (ref. 29) \\
$m_{\mathrm{e}}^{*} / m_{0}$ & 0.196 & 0.212 & $0.20-0.43$ (ref. 30) & $0.03-0.08$ (ref. 26) & $0.22,{ }^{29} 0.2,{ }^{31} 0.21$ (ref. 32)
\end{tabular}

decrease monotonically with increasing biaxial strain. The difference between transverse and longitudinal electron effective mass is very small under tensile strain and can be ignored under compressive strain. Since $\mathrm{BaSnO}_{3}$ is always cubic under hydrostatic strain, the transverse and longitudinal electron effective masses are equal and decrease linearly from compressive hydrostatic strain to tensile hydrostatic strain. The variations of effective mass with volume for $\mathrm{BaSnO}_{3}$, defined as $\mathrm{d} \operatorname{In} m / \mathrm{d} V$, is -2.39 for both $m_{\|}$and $m_{\perp}$, which is larger than that for GaN and AlN reported by Dreyer et al. ${ }^{35}$

To estimate the electron mobility of $\mathrm{BaSnO}_{3}$, we calculated the average electron effective mass for conductivity using the following equation: ${ }^{36}$

$$
\frac{1}{m_{\mathrm{e}}^{*}}=\frac{1}{3}\left(\frac{1}{m_{\|}}+\frac{2}{m_{\perp}}\right)
$$

where $m_{\|}$and $m_{\perp}$ are the longitudinal electron effective mass along the $z$-axial direction and transverse electron effective
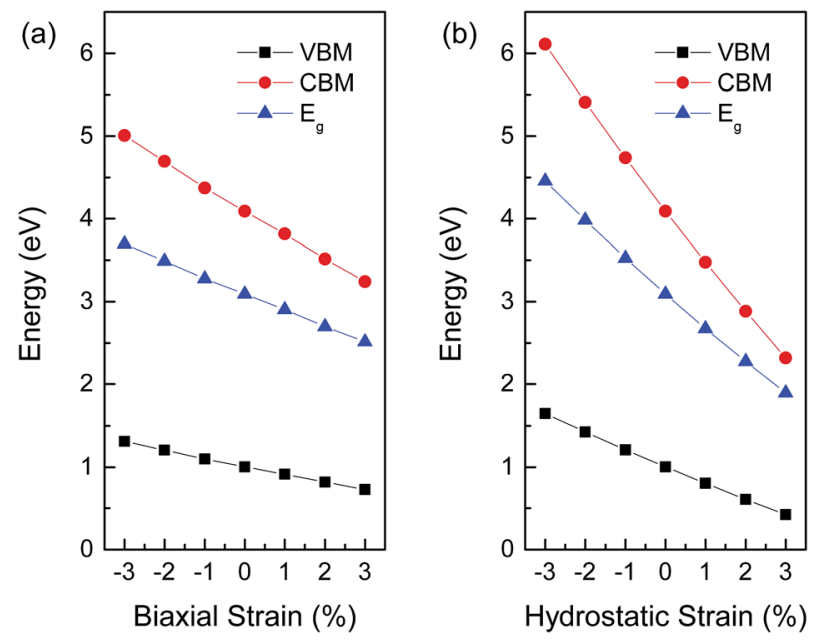

Fig. 3 The position of the conduction band minimum (CBM) and valence band maximum (VBM), and calculated band gap of the $\mathrm{BaSnO}_{3}$ unit cell as a function of (a) biaxial strain, and (b) hydrostatic strain.

Table 2 The deformation potentials of perovskite $\mathrm{BaSnO}_{3}$ under small in-plane biaxial and hydrostatic strain, as defined in eqn (4)

\begin{tabular}{llc}
\hline & $X$ & $D_{\perp}{ }^{X}(\mathrm{eV})$ \\
\hline Biaxial strain & $\Gamma-R$ & -9.99 \\
Biaxial strain & $\Gamma-\Gamma$ & -9.81 \\
Hydrostatic strain & $\Gamma-A$ & -21.36 \\
Hydrostatic strain & $\Gamma-\Gamma$ & -21.45
\end{tabular}

mass along the $x$-axial or $y$-axial directions, respectively. $m_{0}$ represents the effective mass for a free electron. The average electron effective mass of strain-free $\mathrm{BaSnO}_{3}$ using HSE functional theory is $0.212 m_{0}$, which is comparable with the experimental values of $0.20-0.43 m_{0},{ }^{30}$ and the calculated values from other researchers of $0.22 m_{0},{ }^{29} 0.2 m_{0},{ }^{31}$ and $0.21 m_{0}{ }^{32}$ The average electron effective mass decreases linearly under both biaxial and hydrostatic strain. With the increase in strain from $-3 \%$ to $3 \%$, the electron effective mass decreases from $0.25 m_{0}$ to $0.18 m_{0}$ under hydrostatic strain, while it shifts from $0.23 m_{0}$ to $0.20 m_{0}$ under biaxial strain. Thus it is more sensitive to hydrostatic strain than biaxial strain.

The change of electron effective mass with strain can be utilized to estimate the influence of strain on the electron mobility by the equation: ${ }^{37} \mu=e\langle\tau\rangle / m_{\mathrm{e}}^{*}$, with the assumption that $\langle\tau\rangle$ is a constant. $e, m_{\mathrm{e}}^{*}$, and $<\tau>$ are the charge, electron effective mass, and average scattering time, respectively. The normalized electron mobility $\left(\mu / \mu_{0}\right)$ for $\mathrm{BaSnO}_{3}$ under different biaxial and hydrostatic strain with respect to the strain-free $\mathrm{BaSnO}_{3}$ are presented in Fig. 5. $\mu_{0}$ refers to the electron mobility of strain-free $\mathrm{BaSnO}_{3}$. Our results show that the electron mobility is more sensitive to hydrostatic strain than biaxial strain. With the strain change from $-3 \%$ to $3 \%$, the normalized electron mobility monotonously increases from 0.86 to 1.16 under biaxial strain, while increasing from 0.74 to 1.39 under hydrostatic strain. Both tensile hydrostatic and biaxial strain can enhance the electron mobility, while the compressive strain reduces the electron mobility.

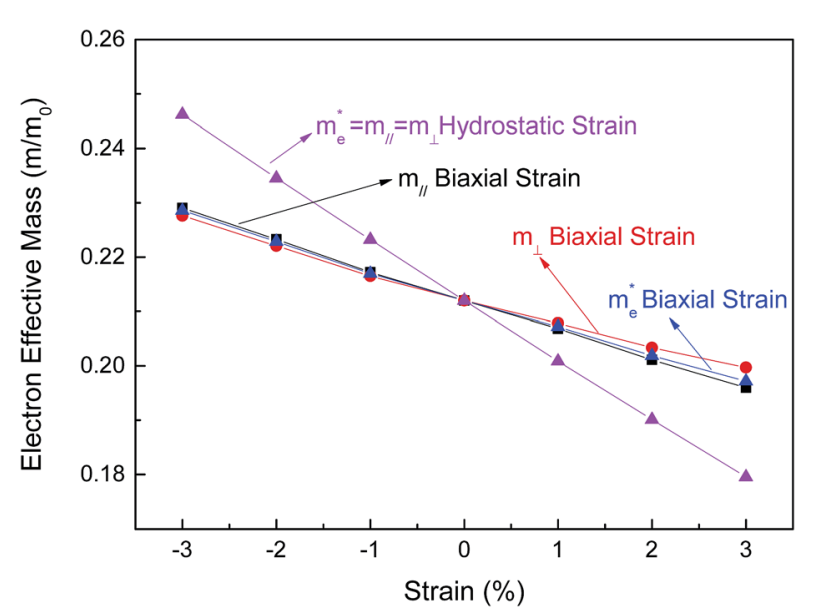

Fig. 4 The dependence of electron effective mass for $\mathrm{BaSnO}_{3}$ under different biaxial and hydrostatic strain. The average electron effective mass for conductivity $\left(m_{\mathrm{e}}^{*}\right)$, longitudinal electron effective mass $\left(m_{\|}\right)$ and transverse electron effective mass $\left(m_{\perp}\right)$ shown in Fig. 1 are presented. $m_{0}$ is the effective mass for a free electron. 


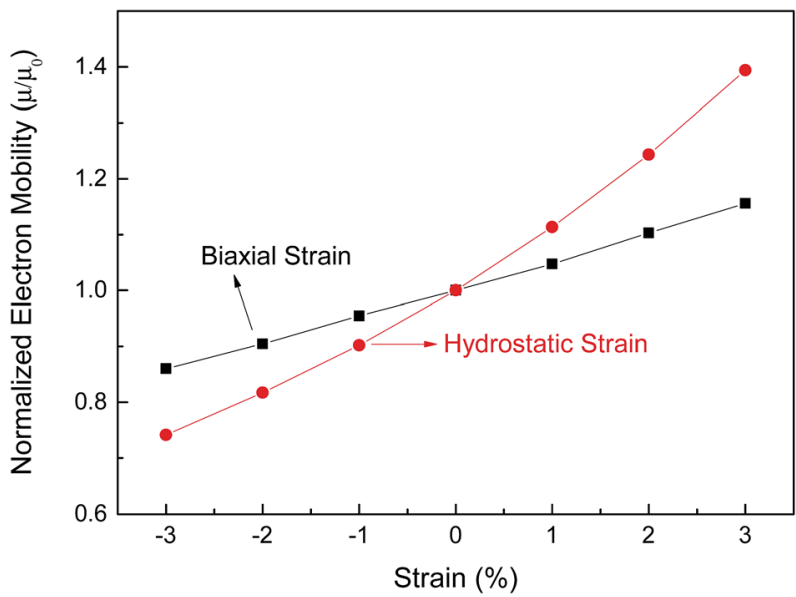

Fig. 5 The normalized electron mobility $\left(\mu / \mu_{0}\right)$ for $\mathrm{BaSnO}_{3}$ under different biaxial and hydrostatic strain with respect to the strain-free $\mathrm{BaSnO}_{3}$.

The variations of band gap and electron mobility compared with the strain-free $\mathrm{BaSnO}_{3}$ as a function of biaxial strain are shown in Fig. 6. The results show that the biaxial strain has opposite effects on the band gap and electron mobility. Compressive strain enhances the band gap and decreases the electron mobility, while tensile strain decreases the band gap and improves the electron mobility. For a change in compressive strain from 0 to $3 \%$, the band gap increases by $19.6 \%$, and the electron mobility drops $13.9 \%$. On the other hand, for tensile strain, $\Delta E_{\mathrm{g}} / E_{\mathrm{g}}$ is negative, and drops linearly with the strain from 0 to $3 \%$. The tensile strain results in a significant improvement of electron mobility, for example, $\Delta \mu / \mu_{0}$ is equal to $15.6 \%$ under $3 \%$ tensile strain.

Further, we expect that the biaxial strains in ultrathin epitaxial $\mathrm{BaSnO}_{3}$ films on suitable substrates can modify the electron mobility and band gap. A number of materials are investigated and some commercially available substrate materials are marked on Fig. 6. It should be pointed out that the

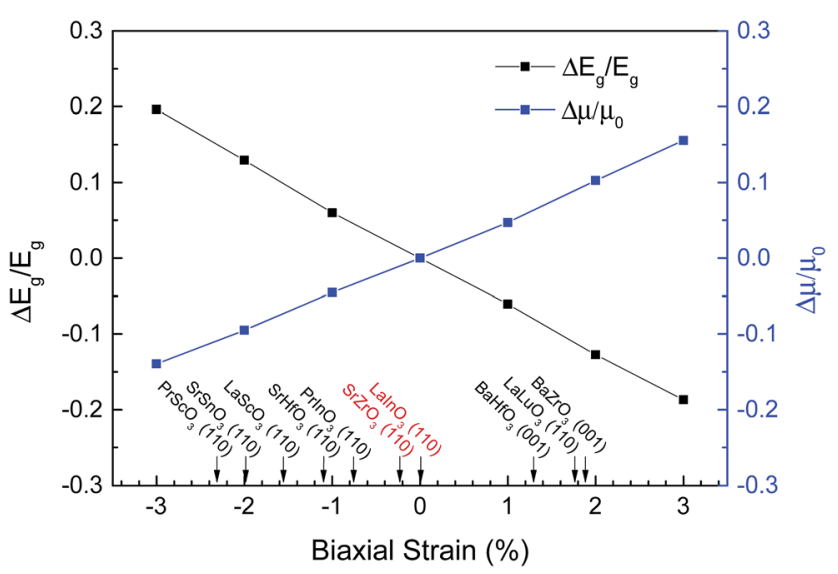

Fig. 6 The variations of band gap and electron mobility of strained $\mathrm{BaSnO}_{3}$ in comparison to the strain-free $\mathrm{BaSnO}_{3}$ as a function of biaxial strain. Some commercially available substrate materials are marked on the figure. substrates marked on the figure only rely on the lattice parameters. However, the strain is not only from the lattice mismatch, but also from the thermal expansion parameters between the film and substrate. In fact, it also depends on the preparation conditions, film thickness, misfit dislocations at the film-substrate interface, and external conditions. The lattice constants of $\mathrm{SrZrO}_{3}$ and $\mathrm{LaInO}_{3}$ are similar to that of $\mathrm{BaSnO}_{3}$, which seems suitable for epitaxial growth to avoid possible misfit dislocations at the film-substrate interface.

\section{Conclusions}

In summary, we have studied the structure, electronic properties, electron effective mass and mobility of perovskite $\mathrm{BaSnO}_{3}$ over a wide range of hydrostatic and biaxial strain using firstprinciples density functional theory calculations. Compared to the biaxial strain, the properties of perovskite $\mathrm{BaSnO}_{3}$ are more sensitive to hydrostatic strain. The structure of $\mathrm{BaSnO}_{3}$ remains cubic for the case of hydrostatic strain, while it changes to tetragonal under biaxial strain. With the change in biaxial and hydrostatic strain from $-3 \%$ to $3 \%$, the band gaps linearly drop from $3.70 \mathrm{eV}$ to $2.51 \mathrm{eV}$, and $4.46 \mathrm{eV}$ to $1.89 \mathrm{eV}$, respectively. The $\mathrm{Sn} 5 \mathrm{~s}$ orbitals of the conduction band minimum play a major role in this strain sensitivity of the band gap. Due to the change of electron effective mass of $\mathrm{BaSnO}_{3}$ under strain, $\mathrm{BaSnO}_{3}$ under tensile strain exhibits higher normalized electron mobility than strain-free $\mathrm{BaSnO}_{3}$, with a value of 1.16 under biaxial strain and 1.39 under hydrostatic strain. On the other hand the compressive strain reduces the normalized electron mobility of $\mathrm{BaSnO}_{3}$ to 0.86 and 0.74 under biaxial and hydrostatic strain, respectively. Hence, the results are a guidance to experimental study, and are also useful to design electronic devices using strained perovskite $\mathrm{BaSnO}_{3}$.

\section{Conflicts of interest}

There are no conflicts to declare.

\section{Acknowledgements}

This work is supported by the National Natural Science Foundation of China (Grant No: 51802268), the Key Laboratory of Fluid and Power Machinery and Young Scholars Support Program of Xihua University, Sichuan Science and Technology Program (Grant No: 2019JDTD0024), and the Yibin Science and Technology Program (Grant No: 2018YG05).

\section{References}

1 A. Prakash, P. Xu, A. Faghaninia, S. Shukla, J. W. Ager III, C. S. Lo and B. Jalan, Nat. Commun., 2017, 8, 15167.

2 Q. Liu, J. Liu, L. Bing, L. Hong, G. Zhu, D. Kai, Z. Liu, Z. Peng and J. Dai, Appl. Phys. Lett., 2012, 101, 241901.

3 H. J. Kim, U. Kim, H. M. Kim, H. K. Tai, H. S. Mun, B. G. Jeon, K. T. Hong, W. J. Lee, C. Ju, K. H. Kim and K. Char, Appl. Phys. Express, 2012, 5, 061102. 
4 S. Raghavan, T. Schumann, H. Kim, J. Y. Zhang, T. A. Cain and S. Stemmer, APL Mater., 2016, 4, 016106.

5 A. Ohtomo and H. Y. Hwang, Nature, 2004, 427, 423-426.

6 H. F. Wang, Q. Z. Liu, F. Chen, G. Y. Gao, W. Wu and X. H. Chen, J. Appl. Phys., 2007, 101, 106105.

7 Q. Liu, J. Feng, G. Gao, L. Bing, Y. Zhang and Q. Liu, J. Alloys Compd., 2016, 684, 125-131.

8 Q. Liu, J. Dai, Z. Liu, X. Zhang, G. Zhu and G. Ding, J. Phys. D: Appl. Phys., 2010, 43, 455401.

9 C. Park, U. Kim, J. J. Chan, S. P. Ji, Y. M. Kim and K. Char, Appl. Phys. Lett., 2014, 105, 203503.

10 J. Shiogai, K. Nishihara, K. Sato and A. Tsukazaki, AIP Adv., 2016, 6, 065305.

11 W. J. Lee, H. J. Kim, E. Sohn, H. K. Tai, J. Y. Park, W. Park, H. Jeong, T. Lee, H. K. Jin, K. Y. Choi and K. H. Kim, Appl. Phys. Lett., 2016, 108, 082105.

12 Z. Lebens-Higgins, D. O. Scanlon, H. Paik, S. Sallis, Y. Nie, M. Uchida, N. F. Quackenbush, M. J. Wahila, G. E. Sterbinsky and D. A. Arena, Phys. Rev. Lett., 2016, 116, 027602 .

13 Y. Zhang, Y. H. Wen, J. C. Zheng and Z. Z. Zhu, Appl. Phys. Lett., 2009, 94, 263116.

14 L. Hong, I. E. Castelli, K. S. Thygesen and K. W. Jacobsen, Phys. Rev. B, 2015, 91, 045204.

15 C. W. Bark, D. A. Felker, Y. Wang, Y. Zhang, H. W. Jang, C. M. Folkman, J. W. Park, S. H. Baek, H. Zhou and D. D. Fong, Proc. Natl. Acad. Sci. U. S. A., 2011, 108, 47204724.

16 Z. Huang, Z. Q. Liu, M. Yang, S. W. Zeng, A. Annadi, W. M. Lü, X. L. Tan, P. F. Chen, L. Sun, X. Renshaw Wang, Y. L. Zhao, C. J. Li, J. Zhou, K. Han, W. B. Wu, Y. P. Feng, J. M. D. Coey, T. Venkatesan and Ariando, Phys. Rev. B: Condens. Matter Mater. Phys., 2014, 90, 125156.

17 K. J. Choi, M. Biegalski, Y. L. Li, A. Sharan, J. Schubert, R. Uecker, P. Reiche, Y. B. Chen, X. Q. Pan and V. Gopalan, Science, 2004, 306, 1005-1009.

18 E. Claude and N. A. Spaldin, Phys. Rev. Lett., 2005, 95, 257601.

19 Y. Umeno, T. Shimada, T. Kitamura and C. Elsasser, Phys. Rev. B, 2006, 74, 174111.
20 H. W. Jang, S. H. Baek, D. Ortiz, C. M. Folkman, R. R. Das, Y. H. Chu, P. Shafer, J. X. Zhang, S. Choudhury and V. Vaithyanathan, Phys. Rev. Lett., 2008, 101, 107602.

21 G. Kresse and J. Furthmuller, Phys. Rev. B, 1996, 54, 1116911186.

22 G. Kresse and D. Joubert, Phys. Rev. B, 1999, 59, 1758-1775.

23 J. Heyd, G. E. Scuseria and M. Ernzerhof, J. Chem. Phys., 2003, 118, 8207-8215.

24 G. Hautier, A. Miglio, D. Waroquiers, G. M. Rignanese and X. Gonze, Chem. Mater., 2014, 26, 5447-5458.

25 P. V. Wadekar, J. Alaria, M. O. Sullivan, N. L. O. Flack, T. D. Manning, L. J. Phillips, K. Durose, O. Lozano, S. Lucas, J. B. Claridge and M. J. Rosseinsky, Appl. Phys. Lett., 2014, 105, 052104.

26 E. Moreira, J. M. Henriques, D. L. Azevedo, E. W. S. Caetano, V. N. Freire and E. L. Albuquerque, J. Solid State Chem., 2012, 187, 186-194.

27 D. O. Scanlon, Phys. Rev. B, 2013, 87, 161201.

28 H. Mizoguchi, P. Chen, P. Boolchand, V. Ksenofontov, P. M. Woodward, C. Felser and P. W. Barnes, Chem. Mater., 2013, 25, 3858-3866.

29 S. Sallis, D. O. Scanlon, S. C. Chae, N. F. Quackenbush, D. A. Fischer, J. C. Woicik, J. H. Guo, S. W. Cheong and L. F. J. Piper, Appl. Phys. Lett., 2013, 103, 042105.

30 S. Chao, T. Huang, J. Zhang, M. Han, Y. Li, Z. Hu and J. Chu, J. Phys. Chem. C, 2014, 118, 6994-7001.

31 H. R. Liu, J. H. Yang, H. J. Xiang, X. G. Gong and S.-H. Wei, Appl. Phys. Lett., 2013, 102, 112109.

32 S. Dabaghmanesh, R. Saniz, M. N. Amini, D. Lamoen and B. Partoens, J. Phys.: Condens. Matter, 2013, 25, 415503.

33 H. Peelaers and C. G. Van de Walle, Phys. Rev. B, 2012, 86, 241401.

34 Q. Yan, P. Rinke, M. Scheffler and C. G. V. D. Walle, Appl. Phys. Lett., 2009, 95, 121111.

35 C. E. Dreyer, A. Janotti and C. G. V. D. Walle, Appl. Phys. Lett., 2013, 102, 142105.

36 F. M. Steel, B. R. Tuttle, X. Shen and S. T. Pantelides, J. Appl. Phys., 2013, 114, 013702.

37 Y. U. Peter and M. Cardona, Fundamentals of Semiconductors: Physics and Materials Properties, Springer Science \& Business Media, 2010. 